\title{
Dispositivo tecnológico para la optimización del tiempo de aprendizaje del lenguaje Braille en personas invidentes
}

\author{
A technological device for optimizing the time taken for blind people \\ to learn Braille
}

Cesar Hernández, Luis F. Pedraza y Danilo López

Universidad Distrital Francisco José de Caldas. Bogotá, Colombia. cahernandezs@udistrital.edu.co, lfpedrazam@udistrital.edu.co dalopezs@udistrital.edu.co

Recibido 20 Febrero 2011/Enviado para Modificación 3 Octubre 2011/ Aceptado 28 Octubre 2011

\section{RESUMEN}

Objetivo El objetivo de este proyecto es diseñar e implementar un prototipo electrónico, que permita mejorar los tiempos de aprendizaje inicial de la escritura Braille en personas con situación de discapacidad visual especialmente en niños. Métodos El desarrollo de este proyecto, se basa fundamentalmente en un prototipo electrónico digital, el cual después de realizar la escritura por parte del usuario, identifica y hace una traducción del Braille por medio de un sistema de síntesis de voz que genera las palabras de manera artificial con el objeto de verificar si la escritura en Braille es o no correcta. Adicional a esto se implementa un módulo GSM, el cual permite enviar mensajes de texto desde el dispositivo, presentando una innovación en el campo de los artículos para personas discapacitadas visualmente.

Resultados El principal resultado obtenido en este proyecto es un prototipo electrónico de fácil acceso y comprensión el cual permite mejorar los tiempos en el aprendizaje inicial de escritura Braille.

Conclusiones Se logró reducir significativamente los tiempos de aprendizaje del lenguaje Braille por parte de personas en situación de discapacidad visual e incrementar su interés y tiempo de concentración en dicho aprendizaje.

Palabras Clave: Aprendizaje, electrónica, lenguaje, personas con discapacidad, tecnología (fuente: DeCS, BIREME).

\section{ABSTRACT}

Objective This project was aimed at designing and putting an electronic prototype into practice for improving the initial time taken by visually handicapped people for learning Braille, especially children.

Methods This project was mainly based on a prototype digital electronic device which identifies and translates material written by a user in Braille by a voice 
synthesis system, producing artificial words to determine whether a handicapped person's writing in Braille has been correct. A global system for mobile communications (GSM) module was also incorporated into the device which allowed it to send text messages, thereby involving innovation in the field of articles for aiding visually handicapped people.

Results This project's main result was an easily accessed and understandable prototype device which improved visually handicapped people's initial learning of Braille.

Conclusions The time taken for visually handicapped people to learn Braille became significantly reduced whilst their interest increased, as did their concentration time regarding such learning.

Key Words: Visually handicapped people, electronics, language, learning, technology (source: MeSH, NLM).

$\mathrm{L}$ a limitación visual se define como la alteración del sistema visual que trae como consecuencia dificultades en el desarrollo de actividades que requieran el uso de la visión (1). Basado en el planteamiento anterior existen dificultades evidentes para las personas en situación de discapacidad visual, entre ellas la imposibilidad de acceder a la información, limitaciones en desarrollo motriz, cognitivo y social o adquirir habilidades en la comunicación.

De acuerdo a cifras publicadas por el DANE en mayo de 2009, existe una población en Bogotá de 64.701 personas con limitación visual, 4.786 personas particularmente en ciudad Bolívar (2). La ley 115 de 1994 (artículo 1) establece que la educación es un proceso de formación permanente, personal, cultural y social que se fundamenta en una concepción integral de la persona humana, de su dignidad, de sus derechos y de sus deberes. Otros artículos de referencia como la ley 361 de 1997 (artículo 10) señala que el Estado Colombiano en sus instituciones de educación pública garantizará el acceso a la educación y la capacitación en los niveles primario, secundario, profesional y técnico para las personas con limitación en sus derechos fundamentales, económicos, sociales y culturales, quienes para ello dispondrán de una formación integral dentro del ambiente más apropiado a sus necesidades especiales (3).

El aporte del presente proyecto consiste en diseñar e implementar una solución tecnológica que permita mejorar los tiempos de aprendizaje del lenguaje Braille en las personas con situación de discapacidad visual especialmente niños. En el mercado nacional actual no existen dispositivos que desarrollen esta función y es por ello que la Biblioteca El Tunal de la ciudad de Bogotá, líder local en la asistencia tecnológica para las personas invidentes debe importar dichos recursos tecnológicos a unos altos costos. Sin embargo, muchos de 
estos recursos son poco utilizados por la tardanza en la capacitación básica del lenguaje Braille. Esta enseñanza inicial del estudiante se hace dispendiosa debido a que es un método lento y personalizado. Estos recursos son limitados para 64.701 personas con limitación visual en Bogotá, lo cual es un obstáculo para disminuir la brecha de inclusión social en el acceso a la comunicación, información y conocimiento de esta población (4).

El proceso inicial de escritura se realiza con una tabla manual de Braille, haciéndose necesario la supervisión de un experto, lo cual limita a la persona en situación de discapacidad visual al no poder ser autodidacta y autónoma en su aprendizaje; es por ello que el presente proyecto plantea una solución tecnológica nacional a esta dificultad (5).

Los resultados obtenidos a partir del dispositivo electrónico desarrollado son bastante satisfactorios en términos del tiempo empleado por la persona invidente y el aumento del interés por aprender el lenguaje Braille (6).

\section{METODOLOGIA}

El proyecto en su totalidad se desarrolló a través de cinco módulos principales como se indica en la Figura1.

- $\quad$ Módulo de Entrada

- $\quad$ Módulo de Control

- $\quad$ Módulo de Almacenamiento

- $\quad$ Módulo de Comunicaciones

- Módulo de Salida

Figura 1. Diagrama de bloques general del diseño

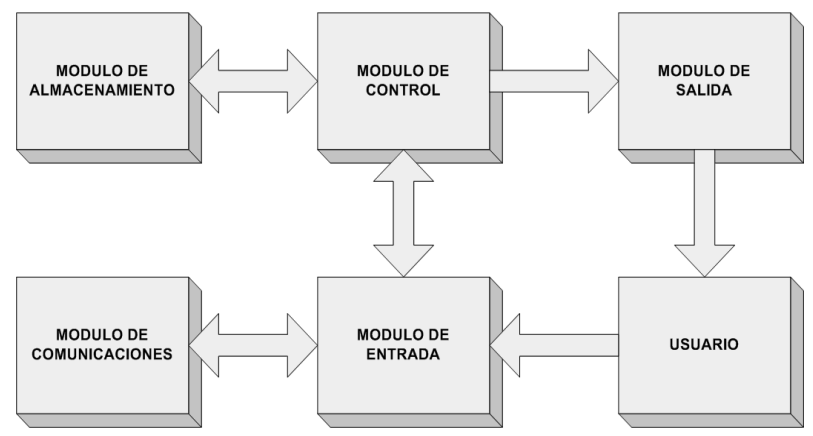


Modulo de Entrada (Teclado)

En el diseño del proyecto se buscó que la implementación del sistema manejara la iteración invidente-sistema y la funcionalidad en el proceso de aprendizaje de escritura Braille.

Para el desarrollo del proyecto se adaptaron elementos funcionales de fácil reconocimiento y maniobrabilidad para el usuario, en este caso la persona con discapacidad visual. Se elaboró un tablero digital, fabricando las celdas Braille de manera manual y verificando que se respetara el estándar de medida utilizado en las regletas Braille, con el objetivo de minimizar las dificultades si fuese necesario cambiar a otra herramienta de aprendizaje.

Estructura Mecánica

El diseño de este sistema consiste en un tablero digital de escritura Braille, conformado básicamente por una estructura donde se encuentra la parte electrónica, una regleta fabricada con los mismos estándares que la utilizada convencionalmente y un punzón de agarre ergonómico, integrado todo en un solo sistema.

La estructura está compuesta por dos laminas de acrílico de $5 \mathrm{~mm}$ de calibre, la lamina superior se encuentra troquelada por orificios en los cuales se encuentran las cajetillas Braille que fueron adaptadas en series de 6 puntos conocidos como símbolo generador Braille, la separación entre cajetillas es $3 \mathrm{~mm}$.

El punzón tiene un agarre ergonómico con base triangular y lados redondeados, que presenta una punta roma metálica para facilidad en los contactos.

\section{Captura de Datos}

El proceso de captura y codificación Braille se realiza mediante el tablero digital. El usuario ingresa la secuencia de pulsos en los símbolos generadores como en un sistema tradicional Braille. La escritura se debe realizar de derecha a izquierda, invirtiendo la numeración de los puntos del cajetín (7).

El modulo de entrada suministra los pulsos eléctricos ingresados por el usuario, los cuales van a un Microcontrolador PIC16F877A, este dispositivo es el encargado de traducir los pulsos recibidos y hacer la equivalencia entre el lenguaje Braille y el código ASCII. 
Cada punto es interpretado como un bit, por lo que el símbolo generador Braille está constituido por seis bits. Se diseña un arreglo matricial de seis bits, haciendo un barrido de cada punto. Si alguna de las 20 columnas (bordes) de la regleta realiza un contacto con el punto, el algoritmo del Microcontrolador le dará la ubicación y la letra. Luego estos datos se almacenan y se envían por el puerto serial.

Modulo de Control

La integración del diseño entre los diferentes módulos, está determinada por el modulo de control, el cual está conformado por un Microcontrolador Microchip PIC 16877A, y los buses de datos que lo comunican con los demás módulos (1). El código de programación ha sido desarrollado en etapas con el fin de verificar cada proceso y obtener el funcionamiento integral de las tareas simultáneas en la identificación de los datos del Módulo de entrada y la coordinación de todo el sistema.

Modulo de voz

El Microcontrolador gestiona y envía la información de forma simultánea al Modulo Sintetizador de Voz (VStamp), para estimular el sentido auditivo. Este Modulo es el encargado de generar la voz de manera artificial y reproducir una señal audible por medio de un parlante. También se puede visualizar la información ingresada por medio de una pantalla de cristal líquido (LCD) para que el proceso de aprendizaje pueda ser monitoreado por una persona vidente que no conozca el lenguaje Braille (8).

Modulo de Comunicaciones

Para la decodificación y control del sistema de comunicación se desarrolla un código que permita interactuar con el hardware (modem). A continuación se detallan las etapas y secuencias de operación.

- El Microcontrolador establece un enlace con el módulo GSM (Enfora GSM0108) por medio del puerto serial (9).

- Una vez establecida la comunicación entre los dos dispositivos se debe programar el Microcontrolador para que transmita los caracteres que forman los comandos AT.

- El PIC siempre está detectando los datos que ingresa el usuario, para ser decodificados y enviados.

- Dependiendo del comando recibido, el PIC ejecutará envío del mensaje. Estas funciones se ejecutan de manera secuencial y ordenada, formando así el programa principal del modulo de comunicaciones. 
Modulo de Almacenamiento

La memoria interna es utilizada como buffer de entrada y salida del módulo de comunicaciones, la memoria externa se utiliza para almacenar los datos que se van a presentar y el mapa de bits con la información que se envía a para visualización y reproducción (10). El Microcontrolador está diseñado para que en su memoria de programa se almacenen todas las instrucciones del programa de control.

La información se almacena en forma de arreglos matriciales realizando la conversión de cada carácter ingresado a código ASCII. El programa se realizó con la finalidad de que el usuario interactúe de forma directa con el sistema, ingresando las palabras o frases que se deseen reproducir o enviar.

\section{RESULTADOS}

Como principal resultado se obtuvo un prototipo electrónico de fácil manipulación a un costo razonable que permite mejorar el tiempo de aprendizaje inicial de la escritura Braille, elevando el grado de autonomía del usuario.

Validación

Las pruebas y validación se realizaron en la sala de Tecnología para discapacitados de la Biblioteca El Tunal y en el Instituto Nacional para ciegos (INCI), con los docentes encargados del área de aprendizaje de la escritura Braille.

Se realizaron un total de diez pruebas con cinco personas en situación de discapacidad visual que se encontraban iniciando su proceso de aprendizaje de escritura Braille. Estas pruebas se realizaron para determinar si el dispositivo corresponde a las necesidades de mejoramiento del aprendizaje.

El trabajo de manipulación del tablero digital permite la familiarización con el signo generador Braille, y el apoyo del sintetizador de voz se hace más fácil conocer, localizar e identificar los puntos del signo generador en el proceso de escritura.

Tiempos de aprendizaje

El tiempo de autonomía en el aprendizaje inicial de escritura Braille se incremento en un $80 \%$ con respecto al sistema tradicional. 
La Tabla 1 presenta los tiempos aproximados de reproducción de SMS con diferentes operadores después de ingresar los caracteres al tablero digital Braille.

Tabla 1. Tiempos de respuesta del prototipo

\begin{tabular}{cc}
\hline Palabra & $\begin{array}{c}\text { Tiempo Aproximado de } \\
\text { Reproducción (segundos) }\end{array}$ \\
\hline Cantante & 1,2 \\
Memoria & 1 \\
Hola & 1,2 \\
Viento & 1,5 \\
Colores & 1,3 \\
Figura & 1,2 \\
Mar & 1 \\
Esperanza & 1,4 \\
Promedio en Tiempo & 1,225 \\
\hline
\end{tabular}

Para medir los tiempos de aprendizaje se conto con la colaboración de los docentes de la Biblioteca El Tunal y el INCI, a través de reuniones periódicas prácticas, llegando los siguientes resultados:

- El tiempo máximo que le toma a un estudiante de Braille escribir un carácter o cajetilla es de 2 minutos.

- El tiempo mínimo promedio que le toma digitar o escribir un carácter es de 2 segundos, alcanzado únicamente por los docentes los cuales gracias a su habilidad adquirida por años de práctica.

Con el prototipo digital se mejora el aprendizaje del lenguaje y la velocidad de digitación ya que el estudiante recibe una confirmación audible cada vez que ingrese un carácter, esto genera un reflejo condicionado y una reacción mas rápida en el momento de expresar una idea.

Porcentaje de error

El porcentaje de error del sistema es del $10 \%$ aproximadamente debido a que se puede presentar una pronunciación no acentuada, es decir, no hay diferencia por ejemplo entre las palabras papa y papá, y la no posible identificación de caracteres especiales.

\section{Costos}

El costo total del primer prototipo fue de $\$ 1165000$ pesos colombianos, sin embargo, el costo marginal de un dispositivo adicional es de tan solo $\$ 380.000$ pesos colombianos. 
Especificaciones Técnicas

La Tabla 2 describe los parámetros técnicos del sistema:

Tabla 2. Parámetros Técnicas del sistema

\begin{tabular}{cc}
\hline Características & Medidas \\
\hline Fuente de alimentación & $5 \mathrm{~V}$ DC y 3,3V DC \\
Potencia de transmisión & $1 \mathrm{~W} \mathrm{GSM} 1800 \mathrm{Mhz}$ \\
Corriente & $0,9 \mathrm{~A}$ \\
Rango de temperatura & 0 a 45 grados \\
Señalización de operación & 6 Leds \\
Monitoreo del sistema & LCD 2x20 \\
Teclado & 20 Cajetines \\
Altura del modulo & $21 \mathrm{~cm}$ \\
Ancho del modulo & $15 \mathrm{~cm}$ \\
Profundidad del modulo & $5 \mathrm{~cm}$ \\
GPS & Antena GPS interna \\
\hline
\end{tabular}

\section{CONCLUSIONES}

El sistema desarrollado facilita el proceso de escritura del lenguaje Braille, con la supervisión de un instructor, se puede obtener un proceso integral donde se conjugan la pedagogía y la tecnología.

El trabajo realizado en la implementación de esta investigación demuestra que es posible desarrollar proyectos que tengan un aporte en la inclusión social de personas en situación de discapacidad visual en el País.

El modulo de comunicaciones funciona en cualquier sitio con cobertura GSM y permite tener una conexión transparente a la red móvil celular mediante la utilización de comandos AT.

Este dispositivo permite que el tiempo de aprendizaje se reduzca en comparación con los métodos tradicionales, permitiendo que la persona en situación de discapacidad visual interactué con el dispositivo de manera sencilla.

La utilización de restos visuales permite un aprendizaje notablemente más rápido con la utilización del sistema

Agradecimientos: Este trabajo de investigación se desarrolló gracias al apoyo de la Universidad Distrital Francisco José de Caldas, el INCI (Instituto Nacional para ciegos), el CRAC (Centro de Rehabilitación para Adultos Ciegos), y la Red Capital de Bibliotecas Públicas. 


\section{REFERENCIAS}

1. Resolución 412 de 2000 [Internet]. Disponible en: http://www.pos.gov.co/Documents/Archivos/ Normatividad_Regimen_Contributivo/resolucion_412_2000.pdf. Consultado 12 de marzo de 2009.

2. Declaración de los Derechos de los Impedidos [Internet]. Disponible en: http://www.inci.gov.co/ info_estadistica.shtml. Consultado 15 de octubre de 2009.

3. Congreso de la república de Colombia [Internet]. Disponible en: http://www.mineducacion.gov.co/ 1621/articles-85906_archivo_pdf.pdf. Consultado 18 de noviembre de 2009.

4. Alegría J, Carrillo M, Sánchez E. La enseñanza de la lectura. Investigación y Ciencia, 2005; 340: 814.

5. Majumder R, Cuervo C. Panorama internacional de la inclusión social de las personas con discapacidad. En: Moreno M. (Ed). Inclusión social de las personas con discapacidad: Reflexiones, realidades y retos. Bogotá DC. 2003; pp. 29-66.

6. Roder B. Percepciones sensoriales de los invidentes. Mente y Cerebro. 2004; 7, 26-29.

7. Aprendizaje Sistema Braille [Internet]. Disponible en: http://usuarios.discapnet.es/ojo_oido/ sistema_braille.htm. Consultado 9 de marzo de 2009.

8. RCsystems. V-STAMP, Synthesizer [Internet]. Disponible en: http://www.rcsys.com/Downloads/ v-stamp.pdf. Consultado 20 de abril de 2009.

9.ModemEnforaGSM0108.[Internet].Disponibleenhttp://www.enfora.com/ index.cgi?CONTENT_ID=2403\&User:LANGUAGE=en. Consultado 2 de marzo de 2010.

10. Microchip. [Internet]. Disponible en: http://ww1.microchip.com/downloads/en/devicedoc/ 39582b.pdf. Consultado 5 de marzo de 2009. 